University of Wollongong

Research Online

Faculty of Social Sciences - Papers (Archive) Faculty of Arts, Social Sciences \& Humanities

2016

The clinical effectiveness of concise cognitive behavioral therapy with or without pharmacotherapy for depressive and anxiety disorders; a pragmatic randomized controlled equivalence trial in clinical practice

\author{
Denise Meuldijk \\ University of Wollongong, meuldijk@uow.edu.au \\ Ingrid V. Carlier \\ Leiden University \\ Irene M. Van Vliet \\ Leiden University \\ T Van Veen \\ Leiden University \\ R Wolterbeek \\ Leiden University
}

See next page for additional authors

Follow this and additional works at: https://ro.uow.edu.au/sspapers

Part of the Education Commons, and the Social and Behavioral Sciences Commons

Research Online is the open access institutional repository for the University of Wollongong. For further information contact the UOW Library: research-pubs@uow.edu.au 


\title{
The clinical effectiveness of concise cognitive behavioral therapy with or without pharmacotherapy for depressive and anxiety disorders; a pragmatic randomized controlled equivalence trial in clinical practice
}

\begin{abstract}
Background: Depressive and anxiety disorders contribute to a high disease burden. This paper investigates whether concise formats of cognitive behavioral- and/or pharmacotherapy are equivalent with longer standard care in the treatment of depressive and/or anxiety disorders in secondary mental health care. Methods: A pragmatic randomized controlled equivalence trial was conducted at five Dutch outpatient Mental Healthcare Centers (MHCs) of the Regional Mental Health Provider (RMHP) 'Rivierduinen'. Patients (aged 18-65 years) with a mild to moderate anxiety and/or depressive disorder, were randomly allocated to concise or standard care. Data were collected at baseline, 3, 6 and 12 months by Routine Outcome Monitoring (ROM). Primary outcomes were the Brief Symptom Inventory (BSI) and the Web Screening Questionnaire (WSQ). We used Generalized Estimating Equations (GEE) to assess outcomes. Results: Between March 2010 and December 2012, 182 patients, were enrolled ( $n=89$ standard care; $n=93$ concise care). Both intention-to-treat and per-protocol analyses demonstrated equivalence of concise care and standard care at all time points. Severity of illness reduced, and both treatments improved patient's general health status and subdomains of quality of life. Moreover, in concise care, the beneficial effects started earlier. Discussion: Concise care has the potential to be a feasible and promising alternative to longer standard secondary mental health care in the treatment of outpatients with a mild to moderate depressive and/or anxiety disorder. For future research, we recommend adhering more strictly to the concise treatment protocols to further explore the beneficial effects of the concise treatment.
\end{abstract}

\section{Keywords}

effectiveness, clinical, concise, behavioral, therapy, without, cognitive, pharmacotherapy, practice, depressive, anxiety, disorders, pragmatic, randomized, controlled, equivalence, trial

\author{
Disciplines \\ Education | Social and Behavioral Sciences
}

\section{Publication Details}

Meuldijk, D., Carlier, I. V. E., van Vliet, I. M., van Veen, T., Wolterbeek, R., van Hemert, A. M. \& Zitman, F. G. (2016). The clinical effectiveness of concise cognitive behavioral therapy with or without pharmacotherapy for depressive and anxiety disorders; a pragmatic randomized controlled equivalence trial in clinical practice. Contemporary Clinical Trials, 47 131-138.

Authors

Denise Meuldijk, Ingrid V. Carlier, Irene M. Van Vliet, T Van Veen, R Wolterbeek, Albert M. van Hemert, and Frans G. Zitman 


\section{The clinical effectiveness of concise cognitive behavioural therapy with or without pharmacotherapy for depressive and anxiety disorders; a pragmatic randomised controlled equivalence trial in clinical practice}

D. Meuldijk ${ }^{\mathrm{a}^{*}}$, I.V.E. Carlier ${ }^{\mathrm{a}}$, I.M. van Vliet ${ }^{\mathrm{a}}$, T. van Veen ${ }^{\mathrm{a}}$, R. Wolterbeek ${ }^{\mathrm{b}}$, A.M. van Hemert ${ }^{\mathrm{a}}$, F.G. Zitman $^{\mathrm{a}}$.

a'Leiden University Medical Center, Department of Psychiatry, Leiden, The Netherlands

${ }^{\mathrm{b}}$ Leiden University Medical Center, Department of Medical Statistics and Bioinformatics, Leiden, The Netherlands

${ }^{*}$ Corresponding author: D. Meuldijk, MSc, Leiden University Medical Center, Department of Psychiatry, Leiden PO Box 9600, 2300 RC, The Netherlands Tel: +31-(0)71-5263785, Fax: +31-(0)71-5248156, e-mail: d.meuldijk@lumc.nl 


\section{Abstract:}

Background Depressive and anxiety disorders contribute to a high disease burden. This paper investigates whether concise formats of cognitive behavioural- and/or pharmacotherapy are equivalent with longer standard care in the treatment of depressive and/or anxiety disorders in secondary mental health care.

Methods A pragmatic randomised controlled equivalence trial was conducted at five Dutch outpatient Mental Healthcare Centres (MHCs) of the Regional Mental Health Provider (RMHP) 'Rivierduinen'. Patients (aged 18-65 years) with a mild to moderate anxiety and/or depressive disorder, were randomly allocated to concise or standard care. Data were collected at baseline, 3, 6 and 12 months by Routine Outcome Monitoring (ROM). Primary outcomes were the Brief Symptom Inventory (BSI) and the Web Screening Questionnaire (WSQ). We used Generalized Estimating Equations (GEE) to assess outcomes.

Results Between March 2010 and December 2012, 182 patients, were enrolled ( $n=89$ standard care; $n=93$ concise care). Both intention-to-treat and per-protocol analyses demonstrated equivalence of concise care and standard care at all time points. Severity of illness reduced, and both treatments improved patients general health status and subdomains of quality of life. Moreover, in concise care, the beneficial effects started earlier.

Discussion Concise care has the potential to be a feasible and promising alternative to longer standard secondary mental health care in the treatment of outpatients with a mild to moderate depressive and/or anxiety disorder. For future research, we recommend adhering more strictly to the concise treatment protocols to further explore the beneficial effects of the concise treatment.

The study is registered in the Netherlands Trial Register, number NTR2590. Clinicaltrials.gov identifier: NCT01643642.

Keywords: Depressive and anxiety disorder; randomized controlled trial; equivalence trial; concise therapy; Routine Outcome Monitoring (ROM); routine secondary mental healthcare 


\section{Introduction}

Depression and anxiety disorders are highly prevalent, cause great suffering and disability, and have a large impact on society (Murphy et al., 2004;Wittchen et al., 2011; Buist-Bouwman et al., 2006; Murray \& Lopez, 1997b; Murray \& Lopez, 1997a). Fortunately, several effective psycho- and pharmacotherapeutic treatments are widely applied for these disorders (Cuijpers et al., 2013). However, they place a high demand on healthcare services (Gustavsson et al., 2011; Nutt, 2011; Smit et al., 2006). Offering these treatments in a more concise form without compromising effectiveness might mitigate this problem (Cape et al., 2010). In this paper, we report the clinical results of a pragmatic, randomized controlled equivalence trial. This entails comparing concise and standard care. We tested the hypothesis thatconcise care is 'as effective as' standard care delivered in a secondary outpatient setting. We focused on patients with mild to moderate illness severity because we assumed that since in most cases their illness is less complicated, they would react more favorable to a concise approach. In both conditions, patients are treated with psycho- and/or pharmacotherapy delivered in routine practice. However, in concise care the treatments are limited in time and in number of (weekly) sessions (maximum 7) and offered as first (brief) step in a stepped-care model (Haaga, 2000; Davison, 2000). Standard care is not confined to a maximum number of sessions or limited time-period (van Fenema et al., 2012). Patient characteristics and treatment effectiveness are assessed with Routine Outcome Monitoring (ROM; de Beurs et al., 2011), a standard monitoring procedure already in use in the participating outpatient clinics. We hypothesized that concise care is equally effective as (equivalent to) standard care 3, 6 and 12 months after baseline assessment. 


\section{Methods}

The methods were published previously (Meuldijk et al., 2012) and are summarized briefly here. The Medical Ethics Committee (MEC) of the Leiden University Medical Center (LUMC) approved the study. It involved a comprehensive protocol (titled “Psychiatric Academic Registration Leiden database”) which safeguarded the anonymity of patients and participants and ensured proper handling of the data. We followed consolidated standards for reporting randomised controlled equivalence trials (Hopewell et al., 2008; Moher et al., 2010; Piaggio et al., 2012). All participants provided written informed consent before study entry.

\subsection{Study design and participants}

A two-armed pragmatic randomised controlled equivalence trial was conducted at five outpatient Mental Health Clinics (MHCs). These clinics were part of Rivierduinen (RD), a secondary Regional Mental Health Provider (RHMP) in the province of South-Holland, the Netherlands. Eligible participants were patients referred to the MHCs by their general practitioners (GP), aged 18-65 years, and meeting the DSM IV-TR criteria for a primary current diagnosis of anxiety disorder and/or depression, established using the MiniInternational Neuropsychiatric Interview-Plus, version 5.0.0 (MINI-Plus; Sheehan et al., 1998; van Vliet \& de Beurs, 2007). For a list of included diagnoses see Appendix A, Table I. Excluded were patients with suicidal or homicidal risk, severe social dysfunction, delusions, hallucinations and/or suffering from bipolar or psychotic disorders. Other comorbidity with psychiatric disorders was allowed. The inclusion (and exclusion) criteria

for enrolling subjects in this study enduced a study sample of patients suffering from mild 
to moderate anxiety and/or depressive disorders (APA, 1994). Insufficient mastery of Dutch was a reason for exclusion. Experienced psychiatrists at the MHCs determined study eligibility (Meuldijk et al., 2012). Eligible participants were randomly assigned to concise or standard care and assessed by ROM at baseline ( $\left.T_{1}\right), 3\left(\mathrm{~T}_{2}\right), 6\left(\mathrm{~T}_{3}\right)$ and $12\left(\mathrm{~T}_{4}\right)$ months thereafter.

\subsection{Randomisation and masking}

A block randomisation scheme, stratified by MHC $(n=5)$ and gender was used.

Randomisation was carried out by one of the researchers (D.M.). Patients and therapists were informed about the outcome; the psychiatric test nurses responsible for the ROM assessments were not (Meuldijk et al., 2012).

\subsection{Treatment}

The treatment protocols in both conditions followed the Dutch and international guidelines for the evidence-based treatment of depressive and anxiety disorders. In both concise and standard care, a choice could be made between pharmacotherapy with a selective serotonin reuptake inhibitor (SSRI; (Guy, 1976), Cognitive Behavioural Therapy (CBT; Clark \& Salkovskis, 1987; Beck, 1995; Clark \& Salkovskis, 1987) and, in case of a posttraumatic stress disorder, Eye Movement Desensitization and Reprocessingtherapy (EMDR; Shapiro, 1995). A combination of pharmaco- and psychotherapy was also possible. In standard care the number of sessions, start and duration of treatment is variable and treatment could continue during the entire study period of 1 year. On average, psychotherapy is provided in 3-6 months on a weekly basis, but in practice once 
every 2 to 3 weeks, pharmacotherapy for 1 year or longer (van Fenema et al., 2012). In contrast, concise care started within one week after the baseline measurement and had to be given within 7 weeks thereafter. Concise care was initially described as 4 to maximum 7 individual 45-minute psychotherapy sessions, depending on the treatment protocol (see also Meuldijk et al. 2012). The pharmacotherapy protocol for depressive and/anxiety disorders in concise care was confined to a maximum of 4 sessions within 7 weeks. Moreover, therapists' treatment choice in both standard and concise care followed the principles of shared decision-making (Hamann et al. 2003; Joosten et al. 2008). Contrary to standard care, treatment goals and procedures in concise care are clearly established and mutually agreed on, prior to initiating treatment. In addition, treatment success of concise care was evaluated at the end of treatment. When either the patient or therapist is convinced that the clinical effects are insufficient or patients are insufficiently helped by the initial treatments in concise care, ‘stepping up’ or continuation of (additional) standard treatment, in line with stepped-care principles, was possible (Haaga, 2000; Davison, 2000). Pharmacotherapy in concise care was also evaluated after 7 weeks, and continued when necessary according to the (inter) national clinical guidelines. After implementation changes to the treatment protocols were made at the recommendation of the MHCs; these included extending the treatment duration of concise care to a maximum of 7 sessions in 7-9 weeks. This was to allow treatment continuation of concise care in case of canceled or missed sessions by therapists or patients.

Therapists providing concise care received a 2 hours instruction in the core elements of the intensified psychotherapy and/or pharmacotherapy, as described in the protocols. 
Therapists in the standard condition did not get additional training (Meuldijk et al., 2012). The same therapists were responsible for delivering standard and concise care. All sessions in concise care were audiotaped for post-hoc assessment of treatment fidelity. Sufficient treatment protocol-adherence (>75\%) was demonstrated in a random sample of 20 patients with a satisfactory overall agreement between two independent raters (Cohen’s Kappa: 0.74). 


\section{Measures}

\subsection{Routine Outcome Monitoring (ROM)}

ROM is a computer-based system to routinely assess symptom severity and functioning with an extensive battery of psychometric instruments. ROM is administered as part of the intake procedure (at baseline) and repeatedly during and after treatment (de Beurs et al.(de Beurs et al., 2011). In the present trial, measures of participants characteristics were collected at baseline, while symptom measures were administered at each time point (see also Meuldijk et al., 2012). An overview of ROM instruments at the different timepoints, is given in Appendix A, Table 2.

\subsection{Outcomes}

The Brief Symptom Inventory (BSI; (Derogatis, 1975) and the Web Screening Questionnaire (WSQ(Donker et al., 2009) constituted the primary outcome measures in this study. The secondary measures used were the Clinical Global Impression (CGI(Guy, 1976) and the Short- Form-36 Health Survey (SF-36; (Ware et al., 1993;Aaronson et al., 1998). Additionally, patients satisfaction with their care was explored by The Dutch Mental Healthcare Thermometer of Appreciation by Clients ((Kok \& van, 2003).

\subsubsection{Primary outcomes}

Brief Symptom Inventory (BSI; (Derogatis, 1975). This patient-rated, 53 item questionnaire which is based on the Symptom Checklist (SCL-90(Derogatis \& Melisaratos, 1983) assesses psychopathological symptom severity on a 5-point Likert 
scale (from 0 'not at all' to 4 'extremely'). The BSI total score, most indicative of general psychopathology, was computed as the mean score of all individual items (range 0-4). Web Screening Questionnaire (WSQ; (Donker et al., 2009). This is a self-rated, 15 item questionnaire which is based on the screening questionnaire (SQ) of Marks and colleagues. It is used as a quick tool to screen patients for most common mental disorders (Gega et al., 2005). The WSQ has 8 'yes' or 'no' answers, the other 7 are Likert-type scales. Response was defined as a score above the pre-specified threshold for being diagnosed with any particular WSQ diagnosis (Donker et al., 2009).

\subsubsection{Secondary outcomes}

Clinical Global Impression (CGI; (Guy, 1976) This is a clinician rated scale that assesses illness severity. The main item 'severity of illness' measured on a 7-point Likert scale (from 1 'normal, not at all ill' to 7 'among the most extremely ill patients') is used in the present analyses.

The Short Form-36 Health Survey (SF-36; (Ware et al., 1993;Aaronson et al., 1998). This self-report questionnaire assesses current general health status and quality of life in eight domains (36 items). Measurement scales vary per subscale, ranging from yes/no to answers on 3-, 5-, or 6-point Likert scale. All raw scores are linearly converted to a 0-100 subscale, with higher scores representing better functioning/quality of life.

In this paper, we report the changes in scores on the three SF-36 subscales which are primarily indicative of physical and mental health (Ware et al. 1994; 2000): physical functioning, social functioning and general health. 
The Dutch Mental Healthcare Thermometer of Appreciation by Clients (Kok \& van, 2003). The thermometer contains 18 items; 17 items with 'yes' or 'no' answers, and one question on 'overall satisfaction with care', (scores from 1 to 5 , higher scores indicating more satisfaction). Two additional items with an open answer format were not included here.

\subsection{Equivalence margins}

The study was designed to demonstrate therapeutical equivalence between concise and standard care and follows study procedures already published in Meuldijk et al. 2012. The sample size calculation (see (Meuldijk et al., 2012) indicated that 500 patients (alpha $=0 \cdot 05,1-\beta=0 \cdot 80$, two-sided) were required to demonstrate equivalence (Jones et al., 1996). Concise care will be deemed equivalent to standard care, if the $95 \%$ confidence interval (CI) for the observed difference in the proportions of treatment success (defined as a 50\% reduction in BSI score compared to baseline BSI score) do not cross these predefined clinical margins of equivalence $(\Delta)$ of $-15 \%$ and $+15 \%$ including an acceptable difference of 5\% (Meuldijk et al., 2012; Wiens, 2002; Jones et al., 1996). The equivalence margins are specified a priori on the basis of a clinical notion of detection, a minimally clinically important difference in effect (see Meuldijk et al.2012). If the treatment effects of concise and standard care differ by more than this equivalence margin in either direction, then equivalence does not hold.

Although not powered for, we similarly evaluated equivalence for WSQ remission (defined as a score below the pre-specified threshold for being diagnosed with any WSQ diagnosis) at 6 and 12 months' measurements. We performed both intention-to-treat 
analyses and sensitivity analyses on the per protocol population (patients who completed both the baseline and the subsequent measurement) to test therapeutic equivalence.

\subsection{Statistical analysis}

We used descriptive statistics for the comparison of the baseline demographic and clinical variables between the groups that completed the treatment (completer group) and the group as randomised; $t$-tests were used for continuous measures and chi-square tests for categorical data. The margins of the $95 \%$ CIs on the basis of the observed difference in the proportions of treatment success of the primary outcomes were compared with the pre-determined equivalence margins. Equivalence was tested in both the intention-totreat and per protocol population.

Additionally, Generalized Estimating Equation (GEE) models (Zeger \& Liang, 1986; Twisk, 2004) were used to estimate the mean differences between treatment groups and the confidence intervals (CIs) at each assessment point for both primary and secondary continuous outcomes; odds ratios with 95\% CIs were given for dichotomous outcomes and differences in means for continuous outcomes. Since our GEE models yields odds ratios as the effect measure instead of risk ratios, effect-sizes are not presented.

For all GEE analyses, the predictor variables were: time (baseline $\left(T_{1}\right)$ as reference, versus $3\left(\mathrm{~T}_{2}\right), 6\left(\mathrm{~T}_{3}\right)$ and 12 month $\left(\mathrm{T}_{4}\right)$ measurement); treatment group (concise care versus standard care), and interaction between time and treatment group. An unstructured correlation matrix was specified in the GEE model to account for the within-patient correlation (repeated, longitudinal observations). 
To investigate if results were overly optimistic due to drop-out of those with less favorable outcome, GEE analyses were repeated with imputation of missing data, by carrying the last observation forward method. Patient satisfaction was explored by between-group comparisons of two sample $t$-tests for continuous variables. GEE analyses for both primary and secondary outcomes were on an intention-to-treat basis using IBM SPSS version 20 (Windows). Significance for all statistical tests was set at a $p$-value $<$ 0.05 (two-sided). 


\section{Results}

Randomisation and treatment allocation took place between March, 2010 and December, 2011. Follow-up assessments were completed for all patients in January, 2013. The passage of the participants through the study is depicted in the CONSORT Flow diagram in Figure 1.

\section{$\rightarrow$ Insert Figure 1 here}

A total of 182 eligible participants provided informed consent and completed the baseline assessment; 93 were randomised to concise care and 89 to receive standard care. $57 \%$ of the participants completed the 3-month assessment $(n=104)$. At assessments $\mathrm{T}_{3}$ and $\mathrm{T}_{4}$, (see Figure 1), respectively $n=65$ and $n=42$ had outcome data.

\subsection{Descriptive statistics}

The socio-demographic and clinical characteristics of the randomised sample and patients who completed both the baseline and the 3-month measurement (completer sample) are summarized in Table 1. Demographic data were available for 178 patients (98\%). The MINI-Plus was administered in 181 patients (99\%).

\section{$\rightarrow$ Insert Table 1 here}

Randomisation reasonably balanced the treatment groups with respect to the baseline characteristics. No statistical differences in baseline clinical or demographic 
characteristics were found between patients who entered the trial and those who dropped out after baseline assessment. These $p$-values ranged from $p=0.061$ to $p=0.892$. In addition, we found no evidence of selective attrition on baseline measures (i.e. primary outcomes) between completers and dropouts (data not shown). According to the MINIPlus interview, 39\% $(n=70)$ of the total sample suffered from depressive (with or without anxiety) disorder and 31\% ( $n=82)$ from anxiety (with or without depressive) disorder(s). Comorbidity between anxiety and depression was found in 17\% (31 patients). 34 patients (19\%) did not pass the threshold for having any current Axis- I DSM-IV-TR diagnosis according to the MINI-Plus interview. These patients did seek professional help for their (subthreshold) depressive and/or anxiety complaints or symptoms.

\subsection{Treatment received}

During the entire study-period, 47 (25\%) of all 182 patients, did not enter treatment (concise care, $n=24$ of 93; standard care, $n=23$ of 89 ). The majority of patients (61\%) started treatment within 3 months after the baseline assessment (concise care, $n=63$ (91\%); standard care $n=48(73 \%)$ ). The majority of these patients were offered psychotherapy (concise care, $n=49$ (78\%); standard care $n=37(77 \%)$ ). Only a small number of patients were offered pharmacotherapy (concise care, $n=4$ (6\%); standard care, $n=6(13 \%)$ ) or a combination of both therapies (concise care $n=10(16 \%)$; standard care $n=5(10 \%))$. In addition, with concise care, the number of therapy sessions (i.e. face to face contacts) was on average as prescribed in the treatment protocols: six sessions for psychotherapy and one for pharmacotherapy. Compared with standard care, the number of therapy sessions did not differ significantly (all P values $>0.05$ ). Patients 
in the standard care group had on average three psychotherapy and one pharmacotherapy sessions (face to face contacts), within the first 3 months after baseline assessment. After the initial 3 months, 18 (27\%) of the patients in standard care and 7 (10\%) of the patients allocated to concise care actually started treatment. The number of patients who remitted or continued treatment after 12 months is unknown. No adverse events were reported.

\subsection{Equivalence: BSI and WSQ}

Table 2 presents the mean differences in the proportions of treatment success of BSI and WSQ and 95\% CIs for both treatment groups over time for the intention-to-treat and the per protocol analysis.

\section{$\rightarrow$ Insert Table 2 here}

The results of Table 2 are visualized in Figure 2. In the intention-to-treat analysis, equivalence of concise and standard care could be demonstrated at $T_{3}$ and $T_{4}$ for the primary outcome measures BSI and WSQ. At assessments $\mathrm{T}_{3}$ and $\mathrm{T}_{4}$ the two-sided 95\% CI's for the difference in the proportions of treatment success for BSI and WSQ scores are within the predetermined equivalence margins $(\Delta)$ of $-15 \%$ and $+15 \%$. Furthermore, at $\mathrm{T}_{2}$, the right margin of the $95 \% \mathrm{CI}$ of the difference in BSI improvement crosses the $\Delta$ margin $(>+15 \%)$ in favor of concise care .

In the per protocol analysis, equivalence of concise and standard care for BSI improvement and WSQ remission has been neither proved or disproved. At $T_{2}, T_{3}$ and $T_{4}$, 
the right margins of the 95\% CI’s for the difference in the proportions of treatment success of the BSI outcome measure cross the $\Delta$ margins $(>+15 \%)$ in the per protocol analysis in favor of concise care. In addition, at $\mathrm{T}_{3}$ and $\mathrm{T}_{4}$ both left and right margins of the $95 \%$ CI of WSQ remission cross the $\Delta$ margins, not establishing equivalence

\section{$\rightarrow$ Insert Figure 2 here}

\subsection{GEE analyses: Primary and Secondary outcomes}

\subsubsection{Primary outcomes}

Figure 3 presents the mean BSI scores and percentage of patients with a psychiatric disorder according to the WSQ at the different assessment points; severity of psychopathology in both treatment groups decreased over time. Furthermore, GEE analyses showed that the difference in BSI scores between groups was the largest 3 months after baseline and in favor of concise care (see Appendix B, Table 1).

\section{$\rightarrow$ Insert Figure 3 here}

\subsubsection{Secondary outcomes}

Mean secondary outcomes scores during treatment for both groups are presented in

Figure 4. The Clinical Global Impression scores reduced over time. Likewise, general health and quality of life improved in both treatment groups. GEE analyses showed statistically significant differences in effectiveness 3 and 12 months after baseline assessment in favor of concise care (see Appendix B, Table 2). 


\title{
$\rightarrow$ Insert Figure 4 here
}

\begin{abstract}
All analyses using imputation with last observation carried forward for the missing data yielded similar results. Since randomization had been successful, we did not explore all possible confounders. Adjusting for age and gender did not influence the differences between the concise and standard care group for any of the primary or secondary outcomes (data not shown).
\end{abstract}

Satisfaction with treatment. Patient satisfaction with treatment was measured 3 months after baseline in 81 patients (missing data in 23 patients). On average, patients in the concise treatment group $(n=41)$ were more satisfied with overall treatment than patients in standard care (mean item difference 1.04; $p=0.024$ ). Satisfaction with the therapist did not differ significantly between groups $(p=0.205)$. 


\section{Discussion}

In this study we demonstrated that a novel 7-sessions concise version of cognitive behavioural therapy and/or pharmacotherapy led to comparable clinical outcomes as the longer standard care in the treatment of depressive and/or anxiety disorders in secondary care outpatients. This was true at the end of concise care at 3 months, but also later at 6 and 12 months and for all effect parameters. In fact, in concise care, the clinical effects are obtained earlier, in the first phase of treatment. Moreover, patients were more satisfied with treatment in concise care.

These results are in line with other studies on the effectiveness of brief treatments for depressive and anxiety disorders (Cape et al., 2010; Nieuwsma et al., 2011; van Straten et al., 2006; Ehlers et al., 2014). Although we cannot state with certainty what underlies the beneficial effect of concise care compared to standard care, we can hypothesize it. Therapists and patients had a central role in treatment. They were asked to strictly following the treatment protocols and actively participate in the study. Besides, prior to starting therapy, treatment goals were formulated and mutually agreed on by both the patient and therapist. These facts, could have also impacted the treatment outcome of concise care. Clinicians and patients could be highly motivated, contributing to this positive treatment effect.

Our study is one of the first to demonstrate these effects in secondary mental health care. Treatment was provided in a regular 'real life' psychiatric outpatient sample by qualified therapists already working in the participating MHCs, reflecting day-to-day clinical practice. This increases external validity, thereby enhancing the generalizability of the results to clinical practice. Moreover, our study has good internal validity as is 
demonstrated by the successful randomization of the treatment groups, the sufficient treatment fidelity and delivery of treatment according to protocols. However, several potential methodological concerns have to be noted. Firstly, the desired sample size of 500 patients was not achieved. An investigation of the inclusion during a 2 month period showed that the majority of patients seeking treatment at the MHCs did not meet the trial inclusion criteria of our study because of the higher severity and burden of disease. Secondly, a substantial number of patients did not complete treatment or were lost to assessment, a common problem in long-term studies in naturalistic out-patient settings (van Noorden et al., 2012; Rush et al., 2006; van Straten et al., 2006). However, although we did not achieve the intended sample size of 500 patients we did find concise care to be at least as effective as concise care. Contrary to our initial expectations and assumptions of a real difference in percentages success between the treatments of $5 \%$ in either direction, the observed difference between the two treatments was larger than expected; e.g. 13\% in favor of concise care in the intention-to-treat population at three months for the primary outcome measure BSI. The observed differences in success percentages at the three time points all being in favor of concise turned out to be sufficient to compensate for the loss to follow-up of patients. Another limitation is that because of the pragmatic study design, patient's suitability to participate in the trial during the course of the study was assessed by the therapists involved. Unfortunately, the reasons for excluding patients from care during the trial were not adequately reported. Due to this lack of knowledge, we cannot make any statements about post-randomisation drop-out and reasons for loss to follow-up after study entry. However, concise care, as such, did not increase dropout rates significantly when 
compared to standard care. In both groups a comparable number of patients had to be excluded, dropped out or did not visit the clinic as scheduled. We found no evidence for selective attrition on baseline characteristics, and our conservative handling of missing data, with last observations carried forward, did not alter the patterns of outcomes. An additional limitation, associated with the pragmatic study design, was that in practice, treatment and therapist adherence to the protocols in concise care was not optimal; not all patients were offered concise care as prescribed in the treatment protocols. Moreover, the design of the study as a RCT in a pragmatic setting resulted in considerable differences in follow-up periods between patients and groups. Although the majority of patients were offered concise care within the first 3 months after study entry, the duration of concise care was prolonged and continued during the entire study period (i.e. therapy sessions in concise care were delivered in 7-9 weeks). However, it appears that concise care did a better job of engaging patients in treatment in a more timely manner. This is reflected in the higher patient satisfaction rating for concise care reported at 3 months. In conclusion, we found that concise care is equally effective as standard care in the secondary outpatient treatment of depressive- and/or anxiety disorders. The early start of concise care, the strict scheduling of weekly patient-therapist contacts and the ongoing monitoring of treatment outcome are important for the beneficial effects of the concise treatment. Since concise care is likely to provide significant health gain, and most beneficial effects are obtained within the first phase of treatment, concise care is recommended as first step in a stepped-care delivery of care. Moreover, in contrast to other studies, concise care examined here follows a stratified stepped-care approach taking into account patients' needs and preferences which may be clinically more 
adequate than the more stringent model of stepped-care which begins with the least intensive treatment for each patient regardless of the patients needs and circumstances. Because the study was carried out in a naturalistic setting, the generalizability of the results is good. The study population reflects the subgroup of lighter cases referred to secondary care, therefore the findings of our study are generalizable to a broader patient population. However, the problems with the inclusion of patients and the high drop-out rate may decrease the generalizability, but these problems are also very difficult to avoid in a naturalistic setting. We hope that future studies with a larger sample size, longer follow-up and more strict adherence to the study protocol, will confirm our promising findings.

\section{Role of funding source}

This is a collaborative study between RD and the department of Psychiatry of the LUMC and is funded entirely by RD. RD is a secondary Regional Mental Health Provider (RHMP) in the province of South-Holland, the Netherlands. The funding source contributed to the study design and enrollment of participants but had no role in data collection, data analysis, data interpretation or writing of the report. The corresponding author had full access to all study data and had final responsibility for the decision to submit for publication.

\section{Conflict of interest}

None of the authors have a conflict of interest related to the findings within the submitted manuscript. 


\section{Contributors' statement}

The funding source, in close collaboration with the LUMC, contributed to the design of the study. Further trial development was carried out by the trial project managers: DM (principal investigator and main author), IVEC (project adviser and co-promotor), IMV (project adviser and co-promotor), AMH (promotor) and FGZ (project adviser and

promotor). Data collection was done by DM, who monitored data collection and integrity of randomisation. The statistical analyses were done by DM. TV and RW provided statistical advice. All authors contributed to the interpretation of the data and drafting of the report, DM was responsible for writing the manuscript. All authors commented on drafts of the manuscript and all approved the final report.

\section{Acknowledgments}

We gratefully acknowledge the funding source Rivierduinen (RD). The authors thank all the patients participating in this study and the staff at RD: psychiatrists, psychologists, psychiatric test nurses, secretary and all others for their contribution to this research. 



\section{References}

Aaronson NK, Muller M, Cohen PD, Essink-Bot ML, Fekkes M, Sanderman R, Sprangers MA, te VA, Verrips E. Translation, validation, and norming of the Dutch language version of the SF-36 Health Survey in community and chronic disease populations. J.Clin.Epidemiol. 1998; 51:1055-1068.

American Psychiatric Association. Diagnostic and Statistical Manual for Mental Disorders- DSM-IV. 4rd ed. Washington, DC: American Psychiatric Association; 1994

Beck JS. Cognitive Therapy: basic and beyond. New York: Guilford Press, 1995.

Buist-Bouwman MA, de GR, Vollebergh WA, Alonso J, Bruffaerts R, Ormel J. Functional disability of mental disorders and comparison with physical disorders: a study among the general population of six European countries. Acta Psychiatrica Scandinavica. 2006; 113:492-500.

Cape J, Whittington C, Buszewicz M, Wallace P, Underwood L. Brief psychological therapies for anxiety and depression in primary care: meta-analysis and meta-regression. BMC.Med. 2010; 8:38.

Clark DM, Salkovskis P.M. Cognitive Treatment for panic attacks: Therapist's Manual. Department of Psychiatry, University of Oxford, 1987.

Cuijpers P, Sijbrandij M, Koole SL, Andersson G, Beekman AT, Reynolds CF, III. The efficacy of psychotherapy and pharmacotherapy in treating depressive and anxiety disorders: a meta-analysis of direct comparisons. World Psychiatry 2013; $12: 137-148$. 
Davison GC. Stepped care: doing more with less? J.Consult Clin.Psychol. 2000; 68:580-585.

de Beurs E, den Hollander-Gijsman ME, van Rood YR, van der Wee NJ, Giltay EJ, van Noorden MS, van der Lem R, van Fenema E, Zitman FG. Routine outcome monitoring in the Netherlands: practical experiences with a web-based strategy for the assessment of treatment outcome in clinical practice. Clinical Psychology and Psychotherapy. 2011; 18:1-12.

Derogatis LR. The Brief Symptom Inventory. Baltimore, MD., Clinical Psychometric Research, 1975.

Derogatis LR, Melisaratos N. The Brief Symptom Inventory: an introductory report. Psychol.Med. 1983; 13:595-605.

Donker T, van SA, Marks I, Cuijpers P. A brief Web-based screening questionnaire for common mental disorders: development and validation. J.Med.Internet.Res. 2009; 11:e19.

Ehlers A, Hackmann A, Grey N, Wild J, Liness S, Albert I, Deale A, Stott R, Clark DM. A randomized controlled trial of 7-day intensive and standard weekly cognitive therapy for PTSD and emotion-focused supportive therapy. Am.J.Psychiatry 2014; 171:294-304.

Gega L, Kenwright M, Mataix-Cols D, Cameron R, Marks IM. Screening people with anxiety/depression for suitability for guided self-help. Cogn Behav.Ther. 2005; 34:16-21.

Gustavsson A, Svensson M, Jacobi F, Allgulander C, Alonso J, Beghi E, Dodel 
R, Ekman M, Faravelli C, Fratiglioni L, Gannon B, Jones DH, Jennum P, Jordanova A, Jonsson L, Karampampa K, Knapp M, Kobelt G, Kurth T, Lieb R, Linde M, Ljungcrantz C, Maercker A, Melin B, Moscarelli M, Musayev A, Norwood F, Preisig M, Pugliatti M, Rehm J, Salvador-Carulla L, Schlehofer B, Simon R, Steinhausen HC, Stovner LJ, Vallat JM, Van den Bergh P, van OJ, Vos P, Xu W, Wittchen HU, Jonsson B, Olesen J. Cost of disorders of the brain in Europe 2010. Eur.Neuropsychopharmacol. 2011; 21:718-779.

Guy W. ECDEU Assessment Manual for Psychopharmacology. National Institue of Mental Health. National Institue of Mental Health, Rockville, MD., 1976.

Haaga DAF. Introduction to the special section on stepped care models in psychotherapy. Journal of Consulting and Clinical Psychology 2000; 68:547-548.

Hopewell S, Clarke M, Moher D, Wager E, Middleton P, Altman DG, Schulz KF. CONSORT for reporting randomized controlled trials in journal and conference abstracts: explanation and elaboration. PLoS.Med. 2008; 5:e20.

Jones B, Jarvis P, Lewis JA, Ebbutt AF. Trials to assess equivalence: the importance of rigorous methods. BMJ 1996; 313:36-39.

Kok I, van WB. Client Appreciation in Mental Health Care: Manual of the Dutch Mental Healthcare Thermometer of Appreciation by Clients [in Dutch]. Utrecht, Trimbos-instituut/GGZ Nederland, 2003.

Meuldijk D, Carlier IV, van Vliet IM, van den Akker-Marle ME, Zitman FG. A randomized controlled trial of the efficacy and cost-effectiveness of a brief intensified cognitive behavioral therapy and/or pharmacotherapy for mood and anxiety disorders: design and methods. Contemp.Clin.Trials 2012; 33:983-992. 
Moher D, Hopewell S, Schulz KF, Montori V, Gotzsche PC, Devereaux PJ, Elbourne D, Egger M, Altman DG. CONSORT 2010 explanation and elaboration: updated guidelines for reporting parallel group randomised trials. BMJ 2010; 340:c869.

Murphy JM, Horton NJ, Laird NM, Monson RR, Sobol AM, Leighton AH. Anxiety and depression: a 40-year perspective on relationships regarding prevalence, distribution, and comorbidity. Acta Psychiatr.Scand. 2004; 109:355-375.

Murray CJ, Lopez AD. Alternative projections of mortality and disability by cause 19902020: Global Burden of Disease Study. Lancet 1997a; 349:1498-1504.

Murray CJ, Lopez AD. Global mortality, disability, and the contribution of risk factors: Global Burden of Disease Study. Lancet 1997b; 349:1436-1442.

Nieuwsma JA, Trivedi RB, McDuffie J, Kronish I, Benjamin D, Williams JW. 2011.

Nutt DJ. The full cost and burden of disorders of the brain in Europe exposed for the first time. Eur.Neuropsychopharmacol. 2011; 21:715-717.

Piaggio G, Elbourne DR, Pocock SJ, Evans SJ, Altman DG. Reporting of noninferiority and equivalence randomized trials: extension of the CONSORT 2010 statement. JAMA 2012; 308:2594-2604.

Rush AJ, Trivedi MH, Wisniewski SR, Nierenberg AA, Stewart JW, Warden D, Niederehe G, Thase ME, Lavori PW, Lebowitz BD, McGrath PJ, Rosenbaum JF, Sackeim HA, Kupfer DJ, Luther J, Fava M. Acute and longer-term outcomes in depressed outpatients requiring one or several treatment steps: a STAR*D report. Am.J.Psychiatry 2006; 163:1905-1917. 
Shapiro F. Eye Movement Desensitization and Reprocessing (EMDR): Basic Principles, Protocols. Guilford Press, 1995.

Sheehan DV, Lecrubier Y, Sheehan KH, Amorim P, Janavs J, Weiller E, Hergueta T, Baker R, Dunbar GC. The Mini-International Neuropsychiatric Interview (M.I.N.I.): the development and validation of a structured diagnostic psychiatric interview for DSM-IV and ICD-10. Journal of Clinical Psychiatry 1998; 59 Suppl 20:22-33.

Smit F, Cuijpers P, Oostenbrink J, Batelaan N, de GR, Beekman A. Costs of nine common mental disorders: implications for curative and preventive psychiatry. Journal of Mental Health Policy and Economics. 2006; 9:193-200.

Twisk JW. Longitudinal data analysis. A comparison between generalized estimating equations and random coefficient analysis. Eur.J.Epidemiol. 2004; 19:769-776. van Noorden MS, Van Fenema EM, van der Wee NJ, van Rood YR, Carlier IV, Zitman FG, Giltay EJ. Predicting outcomes of mood, anxiety and somatoform disorders: the Leiden routine outcome monitoring study. J.Affect.Disord. 2012; 142:122131.

van Straten A, Tiemens B, Hakkaart L, Nolen WA, Donker MC. Stepped care vs. matched care for mood and anxiety disorders: a randomized trial in routine practice. Acta Psychiatrica Scandinavica. 2006; 113:468-476.

van Vliet IM, de Beurs E. The MINI-International Neuropsychiatric Interview. A brief structured diagnostic psychiatric interview for DSM-IV en ICD-10 psychiatric disorders.Tijdschrift voor Psychiatrie 2007; 49:393-397.

van Fenema, E, van der Wee NJ, Bauer M, Witte CJ, Zitman FG. Assessing adherence to 
guidelines for common mental disorders in routine clinical practice.

Int.J.Qual.Health Care 2012; 24:72-79.

Ware JE, Snow KK, Kosinski M. SF-36 Health Survey Manual and Interpretation Guide. Boston: New England Medical Center, The Health Institute, 1993.

Wiens BL. Choosing an equivalence limit for noninferiority or equivalence studies. Control Clin.Trials 2002; 23:2-14.

Wittchen HU, Jacobi F, Rehm J, Gustavsson A, Svensson M, Jonsson B, Olesen J, Allgulander C, Alonso J, Faravelli C, Fratiglioni L, Jennum P, Lieb R, Maercker A, van OJ, Preisig M, Salvador-Carulla L, Simon R, Steinhausen HC. The size and burden of mental disorders and other disorders of the brain in Europe 2010. Eur.Neuropsychopharmacol. 2011; 21:655-679.

Zeger SL, Liang KY. Longitudinal data analysis for discrete and continuous outcomes. Biometrics 1986; 42:121-130. 
Table 1

Socio-demographic and clinical characteristics of the two treatment groups at baseline measurement.

\begin{tabular}{|c|c|c|c|c|c|c|c|c|c|c|c|}
\hline \multirow{3}{*}{$\begin{array}{l}\text { Baseline characteristics } \\
\text { Socio-demographics }\end{array}$} & \multicolumn{2}{|l|}{ Total } & \multicolumn{4}{|c|}{ Randomised Sample } & \multicolumn{5}{|c|}{ Completer sample } \\
\hline & \multicolumn{2}{|c|}{$n=182$} & \multicolumn{2}{|c|}{$\begin{array}{l}\text { Standard care } \\
n=89\end{array}$} & \multicolumn{2}{|c|}{$\begin{array}{l}\text { Concise care } \\
n=93 \\
\end{array}$} & \multicolumn{2}{|c|}{$\begin{array}{l}\text { Standard care } \\
n=52\end{array}$} & \multicolumn{2}{|c|}{$\begin{array}{l}\text { Concise care } \\
n=52\end{array}$} & \multirow[t]{2}{*}{$p$ - value ${ }^{\mathrm{a}}$} \\
\hline & & & & & & & & & & & \\
\hline Age (years): mean (SD) & 36.5 & (12.3) & 37.0 & (11.98) & 36.0 & $(12.0)$ & 37.8 & (11.8) & 38.3 & $(13.1)$ & 0.83 \\
\hline \multicolumn{12}{|l|}{ Gender } \\
\hline Female & 111 & $(61 \%)$ & 53 & $(60 \%)$ & 58 & $(62 \%)$ & 29 & $(56 \%)$ & 31 & $(60 \%)$ & 0.84 \\
\hline \multicolumn{12}{|l|}{ Ethnical background ${ }^{\mathrm{c}}$} \\
\hline Dutch & 167 & $(94 \%)$ & 84 & $(94 \%)$ & 83 & $(94 \%)$ & 50 & $(96 \%)$ & 47 & $(94 \%)$ & 0.96 \\
\hline Other & 10 & $(6 \%)$ & 5 & $(6 \%)$ & 5 & $(6 \%)$ & 2 & $(4 \%)$ & 3 & $(6 \%)$ & \\
\hline \multicolumn{12}{|l|}{ Education level $^{\mathrm{d}}$} \\
\hline Lower education & 68 & $(38 \%)$ & 34 & $(38 \%)$ & 34 & $(39 \%)$ & 19 & $(37 \%)$ & 16 & $(32 \%)$ & 0.78 \\
\hline Higher education & 109 & $(62 \%)$ & 55 & $(62 \%)$ & 54 & $(61 \%)$ & 33 & $(64 \%)$ & 34 & $(68 \%)$ & \\
\hline \multicolumn{12}{|l|}{ Employment status } \\
\hline Employed & 90 & (51\%) & 49 & $(55 \%)$ & 41 & $(47 \%)$ & 25 & (49\%) & 24 & $(48 \%)$ & 0.99 \\
\hline Unemployed/retired & 87 & $(49 \%)$ & 40 & $(45 \%)$ & 47 & $(53 \%)$ & 27 & (52\%) & 26 & $(52 \%)$ & \\
\hline \multicolumn{12}{|l|}{ Marital status } \\
\hline \multirow{2}{*}{\multicolumn{12}{|c|}{ Clinical characteristics/MINI-Plus Diagnosis ${ }^{\mathrm{e}}$}} \\
\hline & & & & & & & & & & & \\
\hline Any Depressive disorder & 70 & $(39 \%)$ & 36 & $(20 \%)$ & 34 & $(19 \%)$ & 22 & $(21 \%)$ & 18 & $(18 \%)$ & 0.49 \\
\hline Any Anxiety disorder & 82 & $(45 \%)$ & 36 & $(20 \%)$ & 46 & $(25 \%)$ & 23 & (22\%) & 29 & $(28 \%)$ & 0.38 \\
\hline Panic disorder (without agoraphobia) & 25 & $(14 \%)$ & 11 & $(6 \%)$ & 14 & $(8 \%)$ & 9 & (17\%) & 8 & $(15 \%)$ & 0.56 \\
\hline Agoraphobia (without panic disorder) & 38 & $(21 \%)$ & 17 & $(9 \%)$ & 21 & $(12 \%)$ & 12 & $(23 \%)$ & 13 & $(25 \%)$ & 0.81 \\
\hline Panic disorder with agoraphobia & 19 & $(10 \%)$ & 8 & $(4 \%)$ & 11 & $(6 \%)$ & 6 & $(12 \%)$ & 6 & $(12 \%)$ & 0.90 \\
\hline Social phobia & 9 & $(5 \%)$ & 3 & $(2 \%)$ & 6 & (3\%) & 1 & $(2 \%)$ & 3 & $(6 \%)$ & 0.78 \\
\hline Specific phobia & 3 & (2\%) & 1 & $(1 \%)$ & 2 & (1\%) & 1 & $(2 \%)$ & 2 & $(4 \%)$ & 0.99 \\
\hline Generalized anxiety disorder & 23 & $(13 \%)$ & 11 & $(6 \%)$ & 12 & $(7 \%)$ & 5 & $(10 \%)$ & 7 & $(14 \%)$ & 0.99 \\
\hline Posttraumatic stress disorder & 15 & $(8 \%)$ & 7 & $(4 \%)$ & 8 & $(4 \%)$ & 2 & $(4 \%)$ & 6 & $(12 \%)$ & 0.42 \\
\hline Obsessive compulsive disorder & 3 & (2\%) & 2 & $(1 \%)$ & 1 & (1\%) & 1 & (3\%) & 0 & $(0 \%)$ & 0.91 \\
\hline Depressive and anxiety disorder & 31 & $(17 \%)$ & 14 & $(8 \%)$ & 17 & (9\%) & 7 & $(14 \%)$ & 10 & $(19 \%)$ & 0.99 \\
\hline No current DSM IV- TR diagnosis & 34 & $(19 \%)$ & 19 & $(22 \%)$ & 15 & $(16 \%)$ & 5 & $(5 \%)$ & 7 & $(7 \%)$ & 0.79 \\
\hline
\end{tabular}

Results are presented as numbers (percentages) for categorical variables and means \pm (standard deviation [SD]) for continues variables. DSM- IV-TR: Diagnostic and Statistical Manual of Mental Disorders; $4^{\text {th }}$ edition. MINI-Plus: mini-international neuropsychiatric interview-plus.

${ }^{a} \mathrm{P}$-values denotes the differences between standard vs. concise care in the completers sample. ${ }^{\mathrm{b}}$ Demographic data; ethnic background, educational status and employment status are missing for 5 participants. ${ }^{c}$ Dutch ethnic background was assumed when the patient and both parents were born in the Netherlands. ${ }^{\mathrm{d} L o w e r}$ education= having completed elementary school, lower general primary education or no education at all; higher education= more than lower education (includes university). ${ }^{\mathrm{e} C l i n i c a l}$ characteristics/diagnosis was missing for 1 participant.

Note. Patients may have more than one MINI-Plus diagnosis, comorbidity was allowed. 
Table 2

Observed differences in the percentages of treatment success over 12 months in the Intention-to-Treat and Per-protocol analysis.

\begin{tabular}{|c|c|c|c|c|c|}
\hline & & \multicolumn{4}{|c|}{ Intention-to-treat } \\
\hline & & Standard care & Concise care & Difference in proportions & $95 \% \mathrm{CI}$ \\
\hline \multirow[t]{3}{*}{ BSI improvement } & $\mathrm{T}_{2}$ & 0.10 & 0.23 & 0.13 & 0.02 to 0.23 \\
\hline & $\mathrm{T}_{3}$ & 0.15 & 0.18 & 0.04 & -0.07 to 0.15 \\
\hline & $\mathrm{T}_{4}$ & 0.11 & 0.16 & 0.05 & -0.05 to 0.15 \\
\hline \multirow[t]{2}{*}{ WSQ remission } & $\mathrm{T}_{3}$ & 0.05 & 0.03 & 0.01 & -0.05 to 0.08 \\
\hline & $\mathrm{T}_{4}$ & 0.06 & 0.05 & 0.00 & -0.08 to 0.08 \\
\hline \multicolumn{6}{|c|}{ Per-protocol } \\
\hline & & Standard care & Concise care & Mean difference & $95 \% \mathrm{CI}$ \\
\hline \multirow[t]{3}{*}{ BSI improvement } & $\mathrm{T}_{2}$ & 0.18 & 0.42 & 0.24 & 0.06 to 0.40 \\
\hline & $\mathrm{T}_{3}$ & 0.38 & 0.63 & 0.25 & -0.00 to 0.46 \\
\hline & $\mathrm{T}_{4}$ & 0.46 & 0.75 & 0.30 & -0.00 to 0.53 \\
\hline \multirow[t]{2}{*}{ WSQ remission } & $\mathrm{T}_{3}$ & 0.15 & 0.16 & 0.01 & -0.20 to 0.25 \\
\hline & $\mathrm{T}_{4}$ & 0.23 & 0.29 & 0.07 & -0.20 to 0.34 \\
\hline
\end{tabular}

Estimated differences in proportions and 95\% CI are presented.

BSI: Brief Symptom Inventory; WSQ: Web Screening Questionnaire. $T_{2}: 3$ month assessment; $T_{3}: 6$ month assessment; $\mathrm{T}_{4}: 12$ month assessment. 


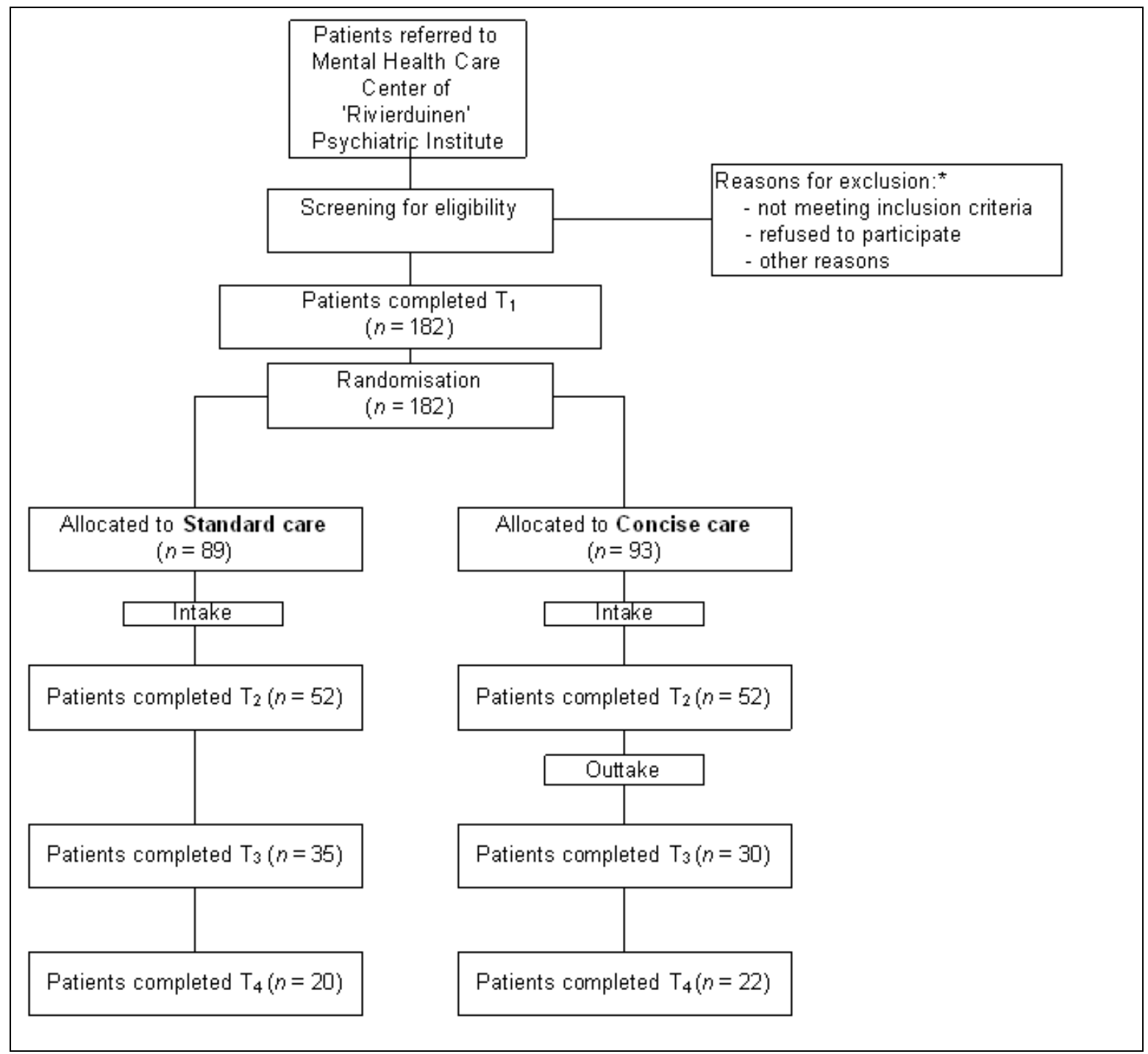

Fig. 1. CONSORT Flow diagram.

T1: baseline assessment; T2: 3 month assessment; T3: 6 month assessment; T4: 12 month assessment.

*Reasons for post-randomisation drop-out after study entry are not known. 


\section{Intention-to-Treat population}

Percentage difference and $95 \% \mathrm{Cl}$

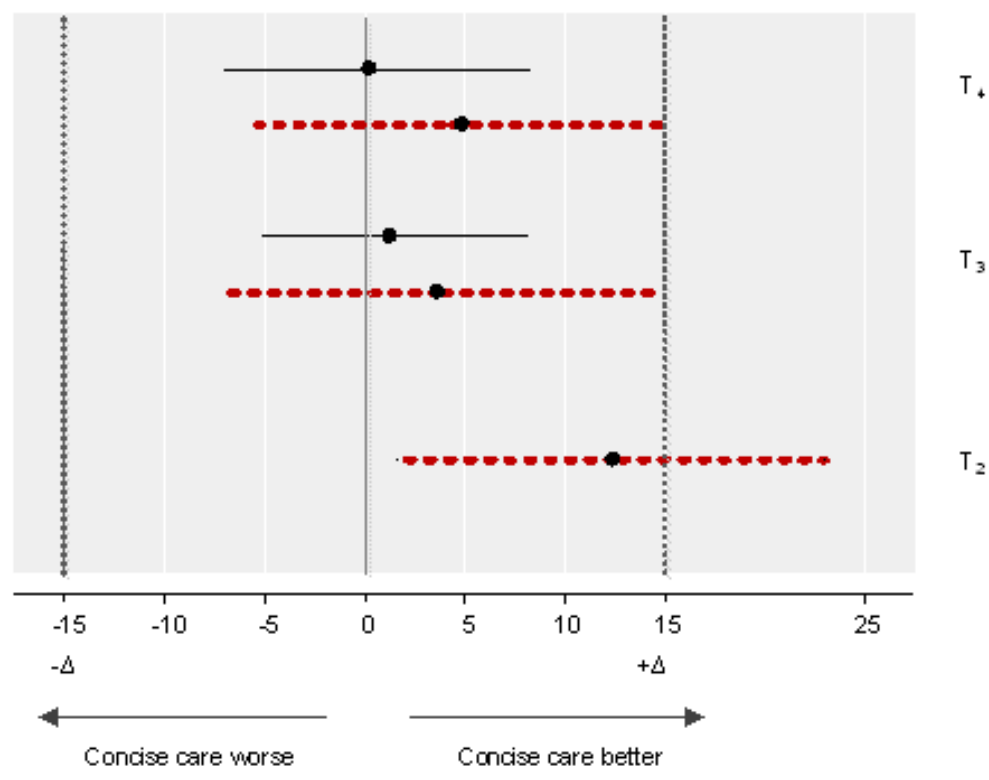

\section{Per protocol population}

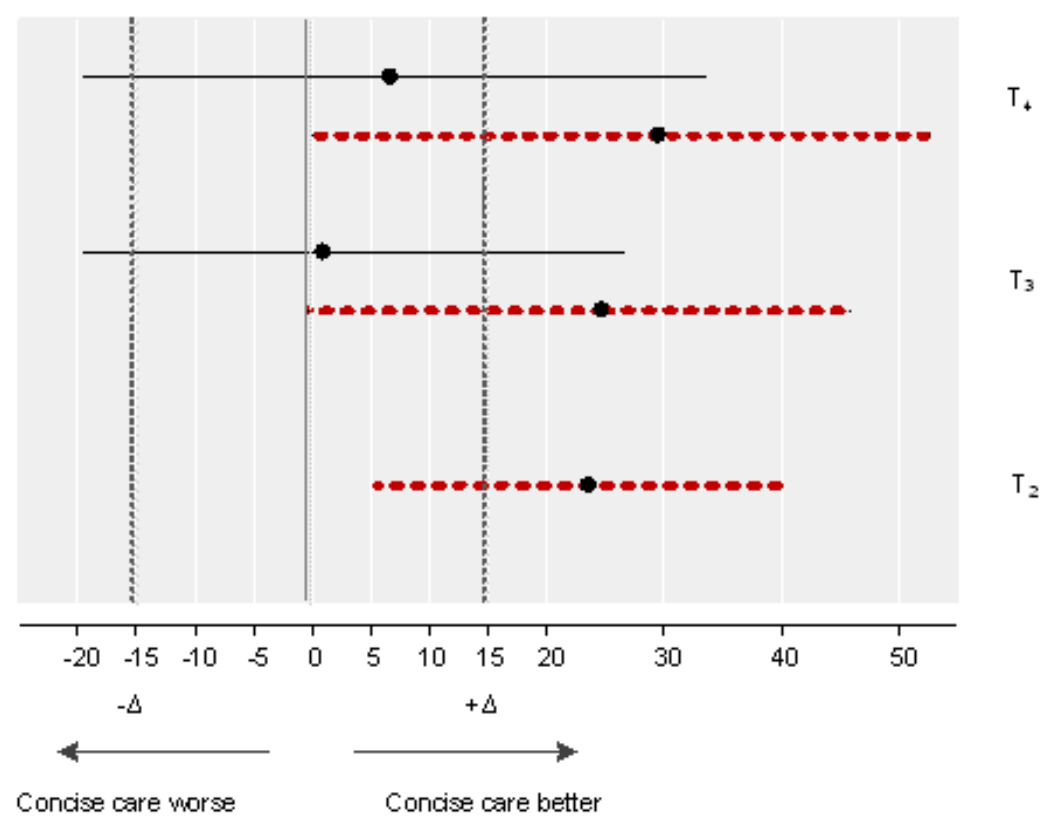

Fig. 2. Equivalence figure BSI and WSQ; Intention-to-Treat analyses vs. Per protocol analyses. Horizontal bars indicate two-sided 95\% confidence interval (CI) of the percentage difference in proportions success between concise care and standard care. The zone between the vertical dashed lines at $\mathrm{x}=-\Delta /+\Delta$ indicates the equivalence margin. BSI: Brief Symptom Inventory; WSQ: Web Screening Questionnaire. $\mathrm{T}_{2}$ : 3 month assessment; $\mathrm{T}_{3}$ : 6 month assessment; $\mathrm{T}_{4}: 12$ month assessment. 


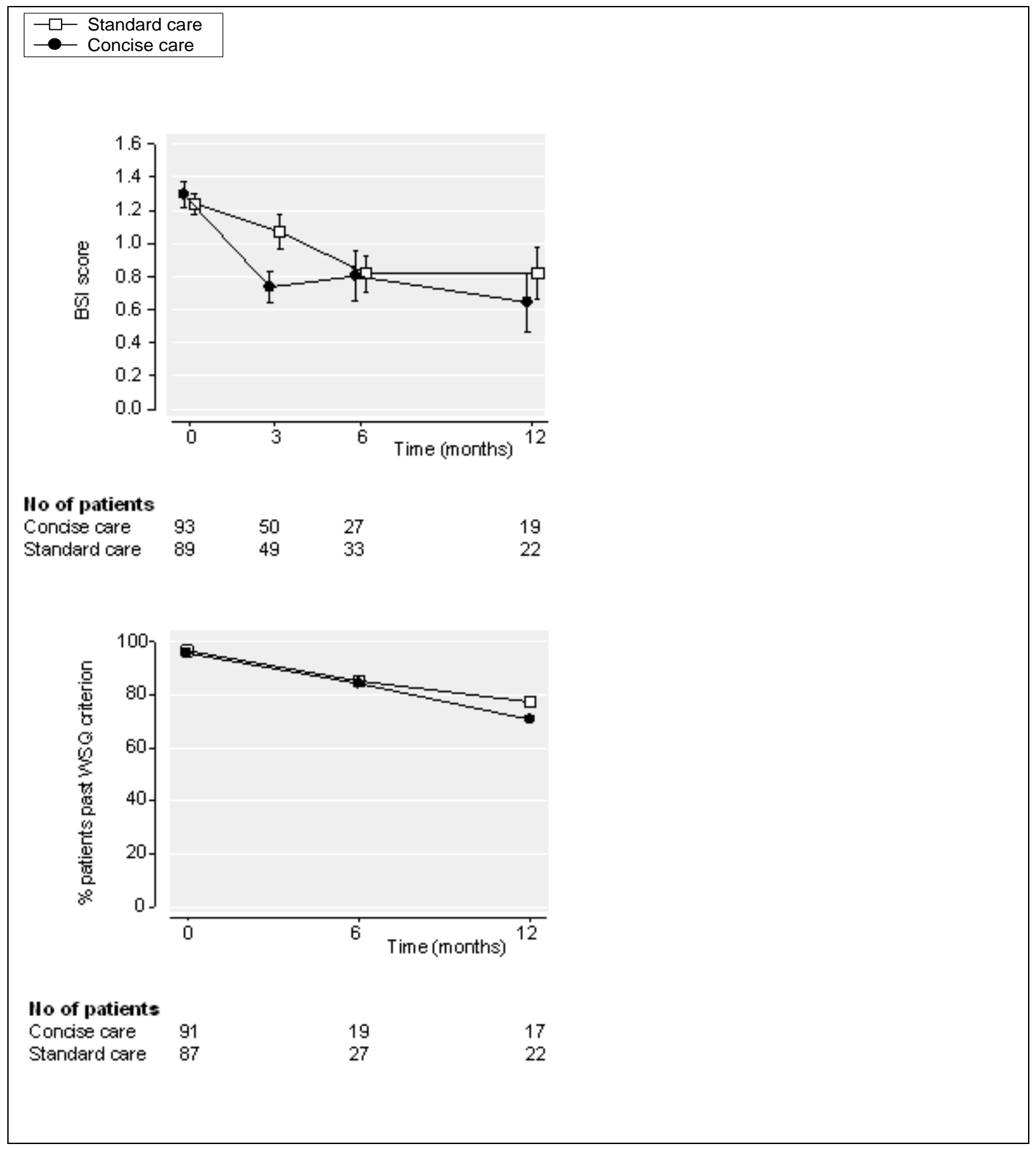

Fig. 3. Observed mean (SE) changes in the primary outcome measure BSI and number of patients being diagnosed with any WSQ disorder in both treatment groups over time.

BSI: Brief Symptom Inventory; WSQ: Web Screening Questionnaire. 


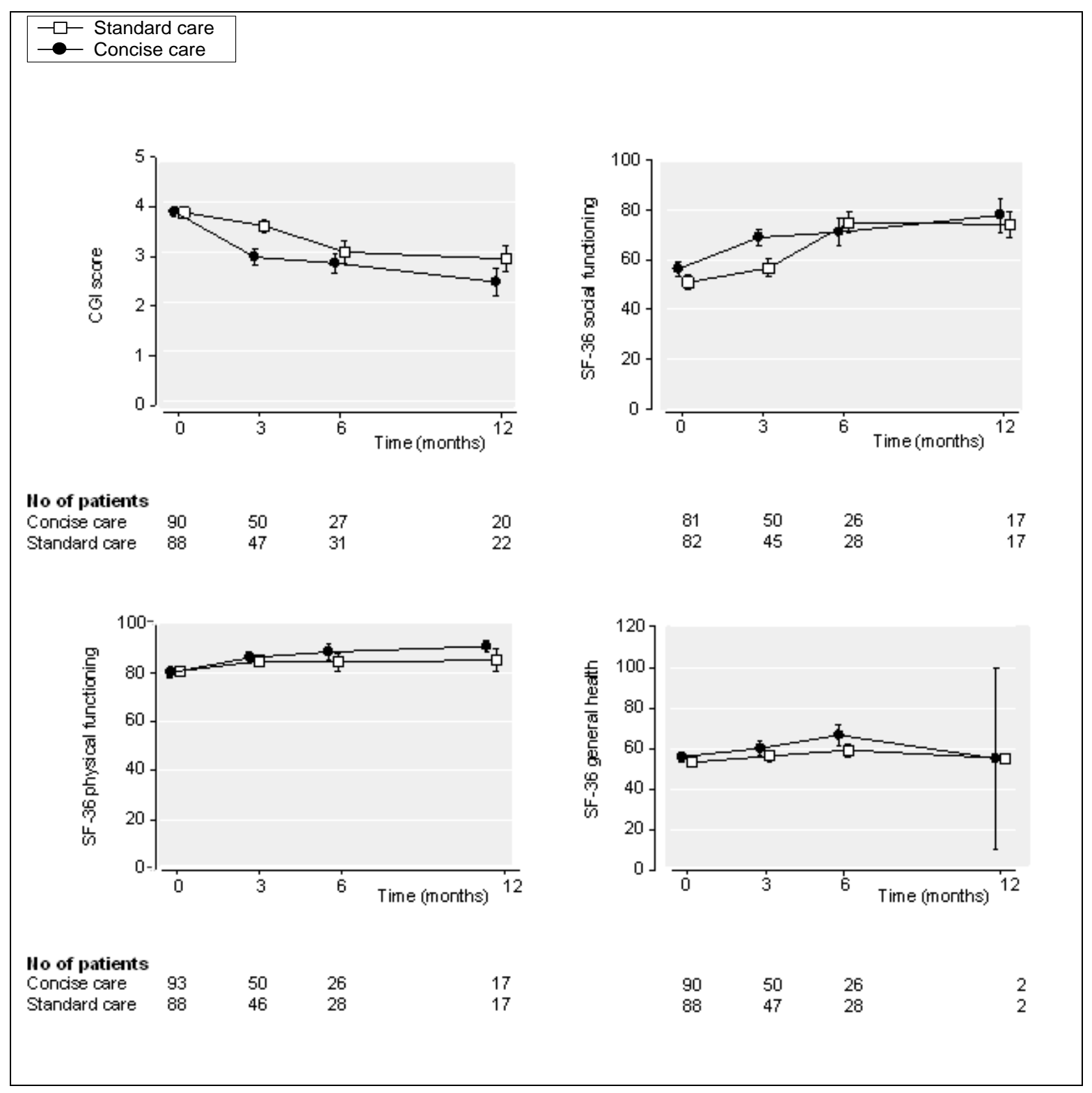

Fig. 4. Observed mean (SE) changes in the secondary outcome measures CGI and SF-36 scores over time in both treatment groups.

CGI: Clinical Global Impression; SF-36: Short-Form Health Survey (SF-36 higher scores representing better functioning/quality of life). 


\section{Appendix A}

Table A. 1

DSM IV-TR diagnoses included within study.

\section{Included DSM IV-TR diagnoses}

minor or major depressive disorder (single episode or recurrent)

depressive disorder NOS

Dysthymia

panic disorder (with or without agoraphobia)

panic disorder NOS

social phobia

simple phobia

generalized anxiety disorder

obsessive compulsive disorder

posttraumatic stress disorder (type I or single trauma),

adjustment disorder (with anxiety and/or depressive mood).

Note: Co-morbidity associated with other psychiatric diagnosis (with the exception of psychotic or bipolar disorder) is allowed.

NOS: Not Otherwise Specified. 


\section{Appendix A}

\section{Table A. 2}

ROM study measures by time interval, in alphabetical order.

\begin{tabular}{|c|c|c|c|c|c|c|c|c|}
\hline Instrument & Full Name & Domain & Cluster $^{\mathrm{a}}$ & Type $^{\text {b }}$ & & e- in & erva & \\
\hline & & & & & $\mathrm{T}_{1}$ & $\mathrm{~T}_{2}$ & $\mathrm{~T}_{3}$ & $\mathrm{~T}_{4}$ \\
\hline BSI & Brief Symptom Inventory & Psychopathology & Gen & SR & $\mathrm{X}$ & $\mathrm{X}$ & $\mathrm{X}$ & $\mathrm{X}$ \\
\hline CGI & Clinical Global Impression & Psychopathology & Gen & OS & $\mathrm{X}$ & $\mathrm{X}$ & $\mathrm{X}$ & $\mathrm{X}$ \\
\hline DEMOG & Demographic Inventory & Social Demographics & Gen & SR & $\mathrm{X}$ & & & \\
\hline Mental Healthcare & Dutch Mental Healthcare Thermometer & Consumer Satisfaction & Gen & SR & & $\mathrm{X}$ & & \\
\hline Thermometer $^{c}$ & of Appreciation by Clients & & & & & & & \\
\hline MINI-Plus & Mini- International Neuropsychiatric Interview Plus & Psychopathology & Gen & OS & $\mathrm{X}$ & & & \\
\hline$S F-36$ & Short Form Health Survey 36 & Psychosocial Functioning & Gen & SR & $\mathrm{X}$ & $\mathrm{X}$ & $\mathrm{X}$ & $\mathrm{X}$ \\
\hline WSQ & $\begin{array}{l}\text { Web Screening Questionnaire for common mental } \\
\text { disorders }\end{array}$ & Psychopathology & Gen & SR & $\mathrm{X}$ & & $\mathrm{X}$ & $\mathrm{X}$ \\
\hline
\end{tabular}

Note: For a list of the complete set of questionnaires used within the study see Meuldijk et al. 2012. In bold: primary and secondary outcome measures. $\mathrm{T}_{1}$ : baseline assessment; $\mathrm{T}_{2}: 3$ month assessment; $\mathrm{T}_{3}: 6$ month assessment; $\mathrm{T}_{4}: 12$ month assessment.

${ }^{\mathrm{a}}$ Gen: Generic cluster. ${ }^{\mathrm{b}} \mathrm{SR}$ : Self Report, OS: Observer Scale. ${ }^{\mathrm{c}}$ Mental Healthcare Thermometer (in Dutch: GGZ Thermometer) .

BSI: Brief Symptom Inventory; CGI: Global Clinical Impression; Demog: Demographics; MINI-Plus: mini-international neuropsychiatric interview-plus; SF-36: Short-Form Health Survey; WSQ: Web Screening Questionnaire. 


\section{Appendix B}

\section{Table B. 1}

Estimated mean differences for the primary outcome measures at baseline, 3 months, 8 months, and 12 months, based on intention-to-treat GEE analysis.

\begin{tabular}{|c|c|c|c|c|}
\hline Measure & Time & Mean difference & $95 \%$ CI & Sig \\
\hline \multicolumn{5}{|l|}{ Primary outcomes } \\
\hline \multirow{4}{*}{ BSI Total score } & Baseline & 0.05 & & \\
\hline & 3 months & -0.30 & -0.54 to -0.06 & $0.01 *$ \\
\hline & 6 months & -0.10 & -0.39 to 0.20 & 0.53 \\
\hline & 12 months & -0.18 & -0.52 to 0.17 & 0.32 \\
\hline & & OR $^{\mathrm{c}}$ & $95 \% \mathrm{CI}$ & \\
\hline \multirow{3}{*}{ WSQ score } & Baseline & 0.78 & & \\
\hline & 6 months & 0.76 & 0.16 to 3.65 & 0.73 \\
\hline & 12 months & 0.78 & 0.17 to 3.71 & 0.80 \\
\hline
\end{tabular}

Estimated mean differences are expressed as $\beta(95 \% \mathrm{CI})$ or as OR $(95 \% \mathrm{CI})$. Standard care is reference category. BSI: Brief Symptom Inventory; WSQ: Web Screening Questionnaire.

*Significant as $\mathrm{p}<0.05$. 


\section{Appendix B}

\section{Table B. 2}

Estimated mean differences for secondary outcomes at baseline, 3 months, 8 months, and 12 months, based on intention-to-treat GEE analysis.

\begin{tabular}{|c|c|c|c|c|}
\hline Measure & Time & Mean difference & $95 \% \mathrm{CI}$ & Sig \\
\hline \multirow{5}{*}{$\begin{array}{l}\text { Secondary Outcomes } \\
\text { Severity of Illness (CGI score) }\end{array}$} & & & & \\
\hline & Baseline & 0.01 & & \\
\hline & 3 months & -0.57 & -0.94 to -0.20 & $0.003 *$ \\
\hline & 6 months & -0.20 & -0.74 to 0.34 & 0.47 \\
\hline & 12 months & -0.35 & -0.98 to 0.28 & 0.28 \\
\hline \multirow{4}{*}{ Social Functioning (SF-36 score) } & Baseline & 1.62 & & \\
\hline & 3 months & 13.35 & 4.28 to 22.42 & $0.004 *$ \\
\hline & 6 months & -3.23 & -14.76 to 8.29 & 0.58 \\
\hline & 12 months & 3.29 & -9.66 to 16.24 & 0.62 \\
\hline \multirow[t]{4}{*}{ Physical Functioning (SF-36 score) } & Baseline & -0.56 & & \\
\hline & 3 months & 0.12 & -5.64 to 5.88 & 0.97 \\
\hline & 6 months & 4.07 & -3.53 to 11.67 & 0.29 \\
\hline & 12 months & 5.01 & -3.87 to 13.89 & 0.27 \\
\hline \multirow[t]{4}{*}{ General Health (SF-36 score) } & Baseline & 1.50 & & \\
\hline & 3 months & 4.21 & -3.54 to 11.96 & 0.29 \\
\hline & 6 months & 5.40 & -3.66 to 14.46 & 0.24 \\
\hline & 12 months & -31.60 & -60.53 to -2.67 & $0.03^{* *}$ \\
\hline
\end{tabular}

CGI: Clinical Global Impression SF-36: Short-Form Health Survey. Standard care is reference category.

* Significant at $\mathrm{p}<0.01$.

$* *$ Significant at $\mathrm{p}<0.05$. 